\title{
Identification of a Functionally Relevant Signal Peptide of Mouse Ficolin A
}

\author{
Sanghoon Kwon', Min-Soo Kim', Dongbum Kim², Keun-Wook Lee ${ }^{2}$, Soo Young Choi ${ }^{3}$, \\ Jinseu Park, Yeon Hyang Kim ${ }^{3}$, Younghee Lee ${ }^{4}$ and Hyung-Joo Kwon ${ }^{1, *}$ \\ ${ }^{1}$ Department of Microbiology and ${ }^{2}$ Center for Medical Science Research, College of Medicine, Hallym University, \\ Chuncheon 200-702, Korea \\ ${ }^{3}$ Department of Biomedical Science, Hallym University, Chuncheon 200-702, Korea \\ ${ }^{4}$ Department of Biochemistry, College of Natural Sciences, Chungbuk National University, Chungbuk 361-763, Korea
}

Received 1 February 2007, Accepted 21 February 2007

\begin{abstract}
Mouse ficolin A is a plasma protein with lectin activity, and plays a role in host defense by binding carbohydrates, especially GleNAc, on microorganisms. The ficolin A subunit consists of an N-terminal signal peptide, a collagen-like domain, and a C-terminal fibrinogen-like domain. In this study, we show that ficolin $A$ can be synthesized and oligomerized in a cell and secreted into culture medium. We also identify a functionally relevant signal peptide of ficolin A by using MS/MS analysis to determine the N-terminal sequence of secreted ficolin A. When the signal peptide of mouse ficolin A was fused with enhanced green fluorescent protein (EGFP), EGFP was released into HEK 293 cell medium, suggesting that the signal peptide can efficiently direct ficolin A secretion. Moreover, our results suggest that the signal peptide of ficolin A has potential application for the production of useful secretory proteins.
\end{abstract}

Keywords: EGFP, Ficolin A, Fusion protein, Secretion, Signal peptide

\section{Introduction}

The immediate recognition of bacteria and of their products by the first line of host defense is mediated by an ancient innate immune response. The innate immune system uses conserved pattern recognition receptors to discriminate between itself and the pathogen-associated molecular pattern signatures of bacterial components (Janeway and Medzhitov, 1998; Aderem and Ulevitch, 2000). In order to elicit an

* To whom correspondence should be addressed.

Tel: 82-33-248-2635; Fax: 82-33-241-3640

E-mail: hjookwon@hallym.ac.kr immune response, the mammalian immune system must be able to recognize a wide variety of bacterial components, including lipopolysaccharide, bacterial DNA, peptidoglycan, lipoteichoic acid, and mannan as representative of an infectious nonself (Aderem and Ulevitch, 2000). The lectin pathway of complement activation is an important component of the innate immune system. The recognition of pathogenassociated molecular patterns in the lectin pathway is mediated by secreted pattern-recognition proteins, such as, mannose-binding lectin (MBL) and ficolins (Matsushita and Fujita, 2001; Holmskov et al., 2003; Roos et al., 2003). These proteins, which have several oligomeric forms, have a carbohydrate-recognition and a collagen-like subunit domain, and are associated through their collagen-like domain with three types of serine proteases termed MBL-associated serine proteases (MASPs), i.e., MASP-1, MASP-2, and MASP-3, which are also complexed by a small MBL-associated protein (Matsushita and Fujita, 1992; Thiel et al., 1997; Dahl et al., 2001). The MBL/ficolins-MASP complexes can recognize pathogens and activate late components of complements, C5C9, which leads to the formation of a cytolytic complex (membrane attack complex) (Thiel et al., 1997; Vorup-Jensen et al., 2000; Rossi et al., 2002; Schwaeble et al., 2002).

Three members of the human ficolin family have been characterized, i.e., L-ficolin (Matsushita et al., 1996) and Hficolin (Sugimoto et al., 1998) - both serum ficolins, and one membrane-associated ficolin expressed in lung and blood cells (Endo et al., 1996). The ficolins (Fujita et al., 2004; Matsushita and Fujita, 2001), which are characterized by the presence of collagen-like domains and fibrinogen-like domains, were primarily identified as transforming growth factor $\beta 1$ binding proteins on porcine uterus membranes (Ichijo et al., 1993) and have lectin activity for $N$-acetylglucosamine (GlcNAc) residues in oligosaccharides (Fujimori et al., 1998; Ohashi and Erickson, 1998; Liu et al., 2005). Researchers have also identified two types of mouse ficolins, ficolin $\mathrm{A}$ and 
ficolin B. Ficolin A is mainly expressed in the liver and spleen and is present in serum, whereas ficolin B is expressed in bone marrow and spleen and has not been detected yet in serum. Endo et al. demonstrated that ficolin A and B share lectin activities for GlcNAc and $N$-acetylgalactosamine, and that ficolin B uniquely recognizes sialic acid residues. Moreover, ficolin A, but not ficolin B, binds MASP-2 and small MBL-associated protein, and the resulting complexes show potent complement-activating capacity (Endo et al., 2005).

In this study, we evaluated HEK 293 cells transfected with an expression vector containing ficolin A cDNA and functionally characterized signal peptide of ficolin A to demonstrate its potential applications for the production of useful secreted proteins.

\section{Materials and Methods}

Plasmid construction. The mouse ficolin A cDNA (1005 bp) was amplified by polymerase chain reaction (PCR) using the mouse macrophage cell line RAW 264.7 cDNA as a template using the following primer sets: 5' primer, 5'-CTCGAGATGCAGTGGCCTA CGCTG-3', and 3' primer, 5'-AAGCTTGATGCTCGGATTTTCAT CTC-3'. The amplified PCR product was ligated into XhoI and HindIII sites of the mammalian expression system vector pcDNA3.1/myc-His(-)B (Invitrogen) to yield the ficolin A expression construct pcDNA3.1-Ficolin A-Myc-His. DNA sequencing was used to verify the nucleotide sequences of the construct.

The signal peptide of ficolin A $(+1$ to +60$)$ was amplified by PCR in which ficolin A cDNA was used as a template, using the following primer sets: 5' primer, 5'-GCTAGCATGCAGTGGCCTA CGCTGTG-3', and 3' primer, 5'-GAATTCCCAGGGCCTGGGAG GGACA-3'. This fragment, which was ligated into NheI and EcoRI sites of pcDNA-3.1-EGFP-myc-His vector, yielded the ficolin A SP (FcnaSP)- EGFP expression vector pcDNA-3.1-FcnaSP-EGFPmyc-His. The sequences of the PCR product and of the in-frame fusion of the signal peptide with the EGFP gene were confirmed by DNA sequencing.

Cell culture and construction of a stable cell line. The human embryonic kidney cell line HEK293 was obtained from the American Type Culture Collection. HEK293 was maintained in Dulbecco's modified Eagle's medium containing 10\% fetal bovine serum, $25 \mathrm{mM}$ HEPES, $100 \mathrm{U} / \mathrm{ml}$ penicillin and $100 \mu \mathrm{g} / \mathrm{ml}$ streptomycin. Cells were cultured at $37^{\circ} \mathrm{C}$ in a $95 \%$ air and $5 \% \mathrm{CO}_{2}$ atmosphere.

To generate a stable cell line, we transfected $5 \mu \mathrm{g}$ of pcDNA3.1-FcnaSP-EGFP-myc-His plasmid, which confers neomycin resistance, into HEK 293 cells using FuGene 6 Transfection Reagent (Roche), according to the manufacturer's instructions. Two days after transfection, cells were selected in $500 \mu \mathrm{g} / \mathrm{ml} \mathrm{G418}$ (Duchefa Biochemie) for 2 weeks. Next, G418-resistant clones were selected and assayed for SP-fused EGFP expression and EGFP secretion by Western blotting and confocal microscopy using a monoclonal antibody against the Myc epitope (Roche). Several positive clones were expanded and analyzed in this study.
Confocal microscopy. FcnaSP-fused EGFP expression was detected by confocal microscopy, as previously described (Kwon et al., 2003). Next, HEK 293 cells $\left(5 \times 10^{4}\right)$ transfected with an FcnsSP-fused EGFP expression vector were cultured directly on glass coverslips in 24-well plates. After $24 \mathrm{~h}$, the cells were fixed with $4 \%$ paraformaldehyde in PBS for $10 \mathrm{~min}$ at room temperature. DNA staining was used to identify cell nuclei $(0.5 \mu \mathrm{g} / \mathrm{ml}$ of Hoechst No. 33258; Sigma). Coverslips were mounted in Fluoromount-G (Southern Biotechnology Associates, Inc.,). The samples were then scanned with a Zeiss LSM 510 laser scanning confocal device attached to an Axiovert 100 microscope with a Plan-Apochromat 100X/Oil DIC objective (Carl Zeiss).

Western blotting. Equal amounts of protein were resolved by $12.5 \%$ SDS-polyacrylamide gel electrophoresis, and electrotransferred to polyvinylidene fluoride membranes (Millipore). The membranes were blocked in Tris-buffered saline containing $0.05 \%$ Tween 20 and $2 \%$ bovine serum albumin for $1 \mathrm{~h}$ at room temperature, and incubated with an anti-Myc antibody for $2 \mathrm{~h}$. Immunoreactive proteins were detected using a horseradish peroxidase-conjugated secondary antibody (Jackson ImmunoResearch Laboratories, Inc.,) and an enhanced chemiluminescence reagent (Amersham Pharmacia Biotech).

Purification of recombinant protein. Recombinant ficolin A was purified from culture supernatant by GlcNAc-agarose (Sigma) affinity chromatography. The culture medium was centrifuged for $20 \mathrm{~min}$ at $15,000 \mathrm{rpm}$ to remove cells and cell debris. All of the following procedures in this experiment were carried out at $4^{\circ} \mathrm{C}$. Samples were loaded onto a GlcNAc-agarose column equilibrated with PBS. The column was washed with PBS until the absorbance at $280 \mathrm{~nm}$ was zero, and recombinant ficolin A was eluted with $0.5 \mathrm{M}$ GlcNAc. To identify the carbohydrate-binding specificities of the ficolin A, we eluted the bound proteins with PBS containing either $0.5 \mathrm{M}$ glucose, $0.5 \mathrm{M}$ galactose, $0.5 \mathrm{M}$ mannose, or $0.5 \mathrm{M}$ GlcNAc. Eluted proteins were resolved by $12.5 \%$ SDS-PAGE under reducing conditions, and analyzed by Western blotting and staining with Coomassie brilliant blue $\mathrm{G}$

To purify Myc-His-tagged protein, culture medium was concentrated with a Centricon YM-50 (Millipore), and the medium removed was replaced with wash buffer $\left(50 \mathrm{mM} \mathrm{NaH}{ }_{2} \mathrm{PO}_{4}, 300 \mathrm{mM} \mathrm{NaCl}, 20\right.$ $\mathrm{mM}$ imidazole, and $\mathrm{pH}$ 8.0). After centrifugation at 15,000 rpm for $20 \mathrm{~min}$ to remove cells and cellular debris, the supernatant was applied to a Ni-NTA-agarose (Qiagen) column pre-equilibrated with wash buffer. After washing the column, the recombinant protein was eluted with elution buffer $\left(50 \mathrm{mM} \mathrm{NaH}_{2} \mathrm{PO}_{4}, 300 \mathrm{mM}\right.$ $\mathrm{NaCl}$, and $250 \mathrm{mM}$ imidazole). Eluted proteins were resolved by $12.5 \%$ SDS-PAGE under reducing conditions and analyzed by Western blotting using anti-Myc antibody.

N-terminal amino acid sequence analysis. The ficolin A band derived from culture medium was resolved by $12.5 \%$ SDSpolyacrylamide gel electrophoresis and stained with Coomassie brilliant blue G (Sigma). To determine the N-terminal amino acid sequence of the secreted ficolin A, we ensured the protein band was digested in gel with trypsin and then purified the resulting peptides using a POROS R2 column (Applied Biosystem) to concentrate and desalt the sample. We then analyzed the samples by ESI-TOF 
MS/MS using a Micromass Q-TOF MA equipped with a nanospray source (Waters, Milford) in an In2gen facility. The resulting amino acid sequences were analyzed using the database managed by the National Center for Biotechnology Information (http://www.ncbi. nlm.nih.gov).

\section{Results and Discussion}

Oligomerization and secretion of recombinant ficolin $\mathbf{A}$. To determine whether mouse ficolin A could be oligomerized and secreted by mammalian cells, we cloned full-length mouse ficolin A cDNA into a pcDNA-3.1/myc-His(-)B vector to produce a construct containing an expression cassette for ficolin A and a neomycin-resistance cassette for G418 selection. The expression plasmid was introduced into HEK 293 cells using FuGene 6 transfection reagent, and transfected cells were selected using G418. Western blotting was then used to analyze the oligomeric composition of ficolin A. Using SDS-PAGE analysis under non-reducing conditions, we found that ficolin A was a mixture of monomers and oligomers and that the mixture migrated as multiple discrete bands in conditioned medium and in cell lysates (Fig. 1). Figure 1 also suggests that the ficolin $\mathrm{A}$ is synthesized and oligomerized in cells and secreted into culture medium. As expected, ficolin A was identified as a single band when the protein was analyzed under reducing conditions.

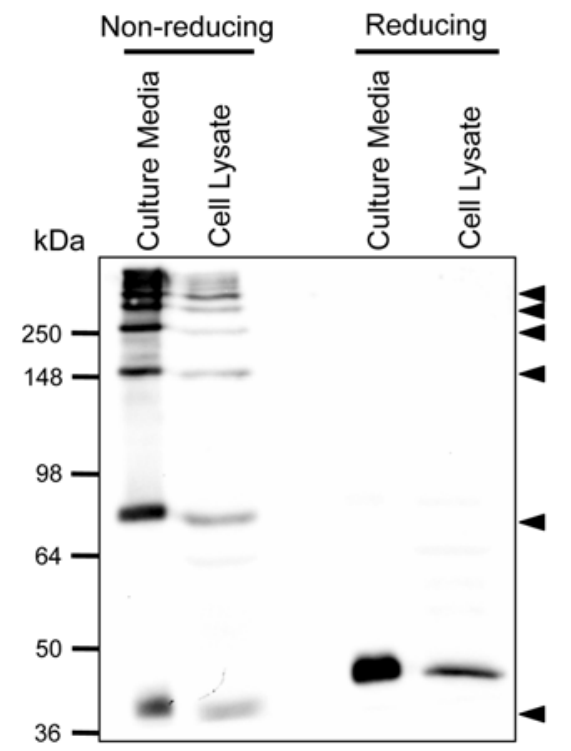

Fig. 1. Oligomerization and secretion of recombinant ficolin A. HEK 293 cells were transfected with ficolin A expression vector and stable cell lines were selected with G418. Protein samples of stable cell lysates and culture medium were electrophoresed $(8 \%$ SDS-PAGE) under non-reducing or reducing conditions. The expression and secretion of ficolin A were analyzed by Western blotting using antibody against Myc. The positions of monomeric and oligomeric ficolin A are indicated by arrowheads.
Purification and carbohydrate-binding specificities of recombinant ficolin A protein. Because ficolins bind GlcNAc (Endo et al., 2005), we purified the recombinant ficolin A using affinity chromatography using a GlcNAcagarose column from the culture medium of HEK 293 cells expressing Myc-His-tagged ficolin A. The secreted ficolin A bound to the GlcNAc column and was eluted by $0.5 \mathrm{M}$ GlcNAc (Fig. 2). As shown in Fig. 2A, the eluted ficolin A was identified as a single band of $39 \mathrm{kDa}$ when subjected to SDS-PAGE under reducing conditions and was stained by Coomassie brilliant blue G. The secreted ficolin A band was also detected using an anti-Myc antibody (Fig. 2B).

To identify the carbohydrate-binding specificities of the secreted ficolin A, we treated the protein bound to the GlcAcagarose column with PBS containing either $0.5 \mathrm{M}$ glucose,
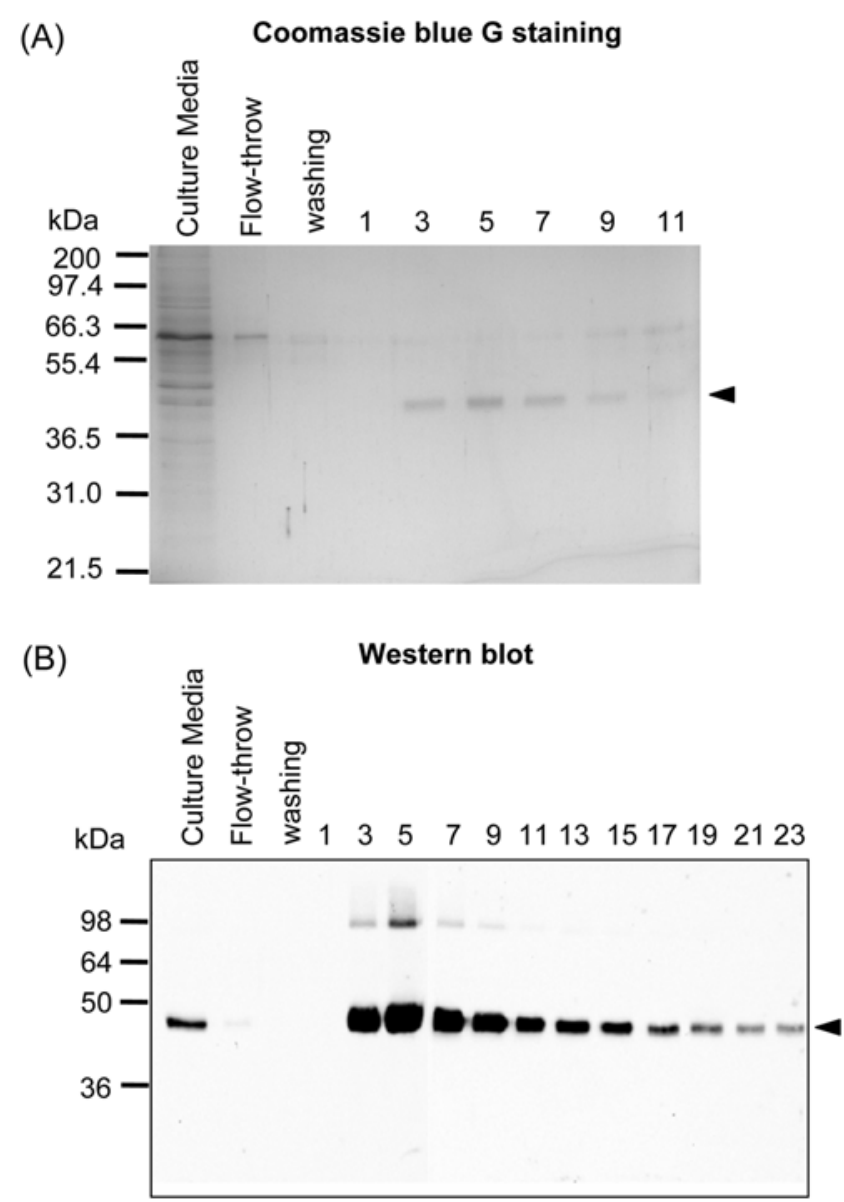

Fig. 2. Purification of recombinant ficolin A by GlcNAc affinity chromatography. Culture medium of the ficolin A transfected stable cell line was loaded onto a GlcNAc-agarose column and eluted with 0.5 M GlcNAc. Samples from each eluted fraction were analyzed by SDS-PAGE $(12.5 \%)$ under reducing conditions. Gels were stained with Coomassie blue G (A). Ficolin $\mathrm{A}$ in each eluted fraction was analyzed by Western blotting using antibody against Myc (B). The positions of secreted ficolin A are indicated by arrowheads. 


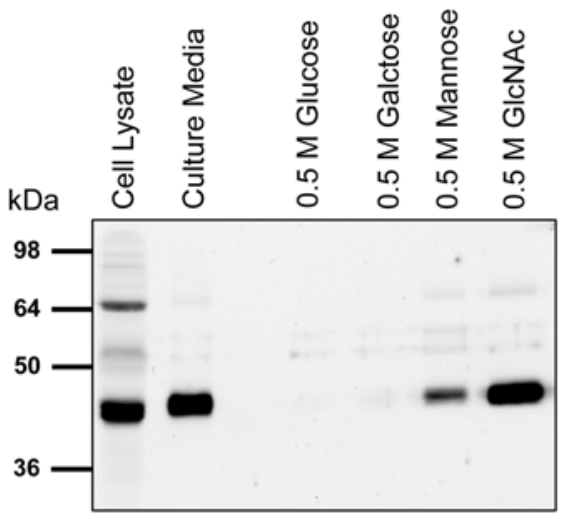

Fig. 3. Carbohydrate-binding specificities of recombinant ficolin A. Culture medium of the ficolin A transfected stable cell line was loaded onto a GlcNAc-agarose column and eluted with either $0.5 \mathrm{M}$ glucose, $0.5 \mathrm{M}$ galactose, $0.5 \mathrm{M}$ mannose, or $0.5 \mathrm{M}$ GlcNAc. Eluted ficolin A was analyzed by Western blotting using antibody against Myc.
0.5 M galactose, 0.5 M mannose, or 0.5 M GlcNAc. As shown in Fig. 3, the secreted ficolin A was fully eluted by GlcNAc. However, ficolin A was only partially eluted by mannose, suggesting its weaker binding to mannose. These specificities are similar to those of the previously identified ficolin A expressed by Drosophila S2 cells (Endo et al., 2005).

N-terminal amino acid sequence analysis of secreted ficolin A. To determine the N-terminal amino acid sequence of the secreted ficolin A, we ensured that the protein band was digested in gel by trypsin and then analyzed the peptides produced by MS/MS Q-TOF. The resulting fragments of each peak were consistent with the amino acid sequence of ficolin $A$ in the NCBI database of MALDI-TOF fingerprints. The MS/MS Q-TOF 779.9078 peak was identified as a SQAL GQERGACPDVK, corresponding to residues 18-32 of fulllength ficolin A. Ficolin A fragments containing further upper amino acid residues could not be detected by MS/MS (Fig. 4A). The above results indicate that mouse ficolin A contains

(A)

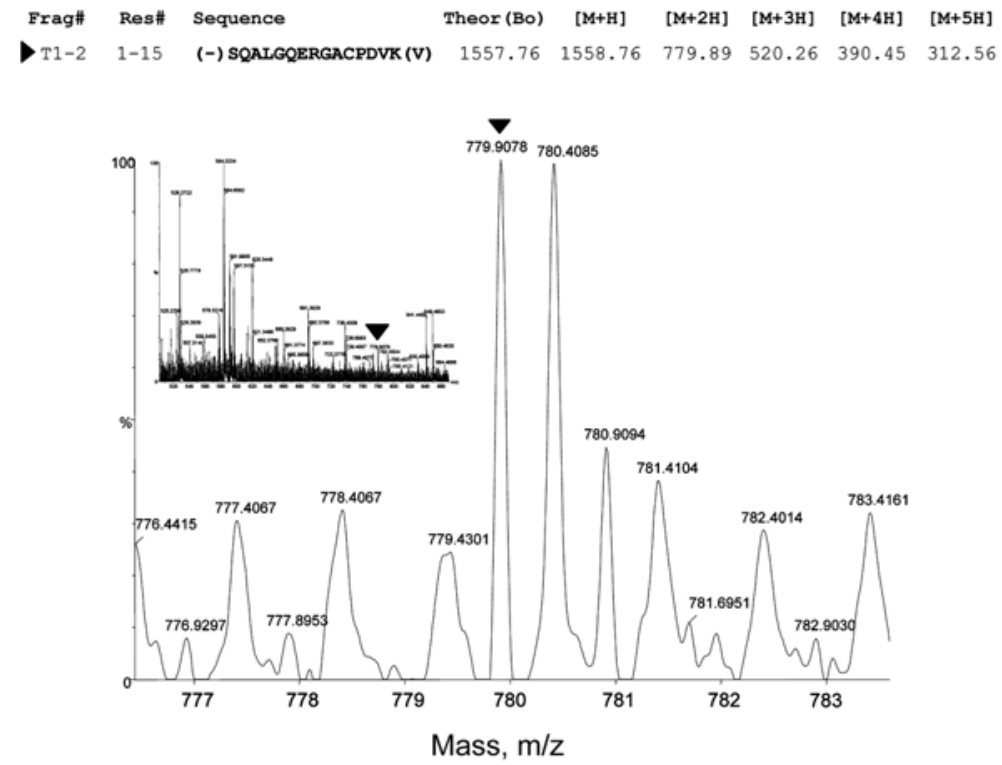

(B)

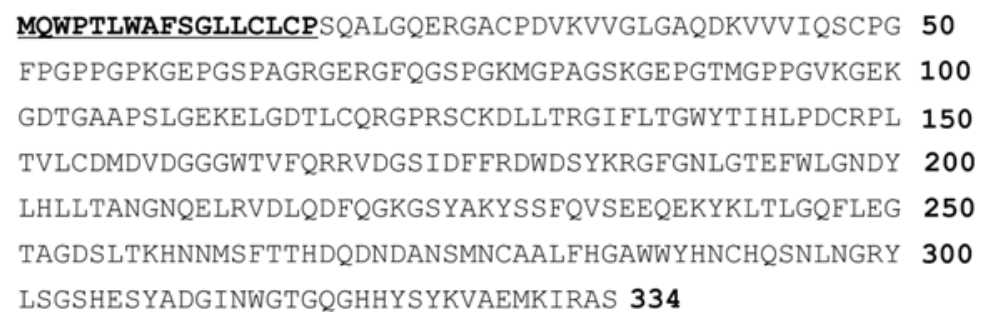

Fig. 4. MS/MS spectra and the determination of the sequence of ficolin A signal peptide. (A) A secreted ficolin A band was digested in gel with trypsin, and the sample obtained was analyzed by ESI-TOF MS/MS. MS/MS analysis of the 779.9078 mass peak (arrow) revealed the N-terminal sequence of the secreted ficolin A. (B) The amino acid sequence of mouse ficolin. The signal peptide is thickly underlined. 
a short N-terminal signal peptide sequence comprised of seventeen amino acids, MQWPTLWAFSGLLCLCP (Fig. 4B).

Use of the signal peptide of ficolin A to secrete EGFP. To verify the function of the signal peptide of mouse ficolin A in terms of protein secretion by mammalian cells, we used PCR to isolate the DNA sequence encoding the first 17 amino acid residues from ficolin A (FcnaSP) identified in this study, and then fused this DNA sequence to full-length EGFP protein (Fig. 5A). The FcnaSP-EGFP gene was cloned into a pcDNA 3.1-Myc-His vector under the transcriptional control of CMV promoter used for mammalian cell expression studies (Lee et al., 2004).

Next, HEK 293 cells were transfected with pcDNA-3.1FcnaSP-EGFP-myc-His vector, and stable cell lines were isolated by selecting G418-resistant clones. As shown in Fig. $5 \mathrm{~B}$, FcnaSP-EGFP fusion protein was detected in stable cell lines by confocal microscopy. In contrast, EGFP protein expression in control cells transfected with empty vector was not detected.

To purify the secreted FcnaSP-EGFP fusion protein, we harvested culture medium. Culture medium was concentrated with Centricon YM-50 and applied to a Ni-NTA-agarose column, and the recombinant protein was eluted. Eluted proteins were analyzed by Western blotting using anti-Myc antibody and EGFP was detected (Fig. 5C). Our results indicated that EGFP was secreted into HEK 293 cell medium under the control of the signal peptide of ficolin A.

Extracellular proteins are constitutively or inducibly secreted by cells. Protein secretion mediated by a co-translational transport system generally involves $\mathrm{N}$-terminal signal peptides. After being translated on rough endoplasmic reticulum (ER), proteins are secreted via a process known as the ER/Golgidependent secretory pathway. Nascent proteins scheduled for

(A)

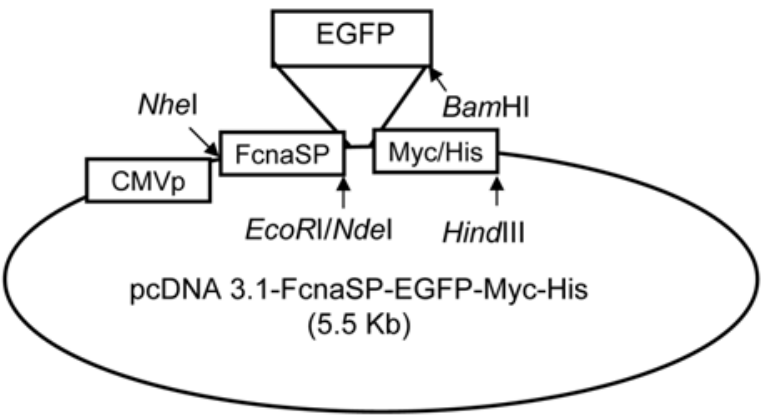

(B)
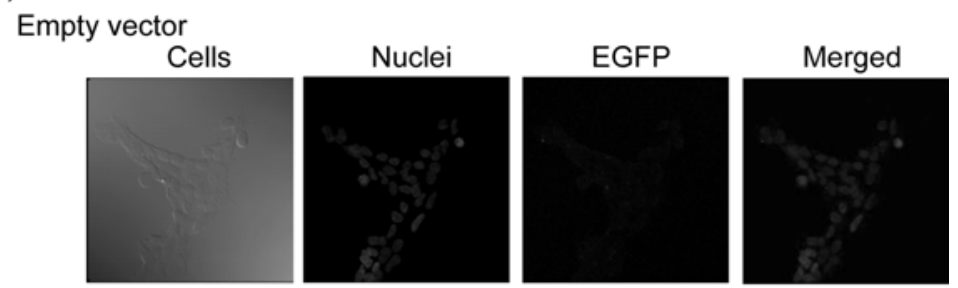

pcDNA-FcnaSP-EGFP
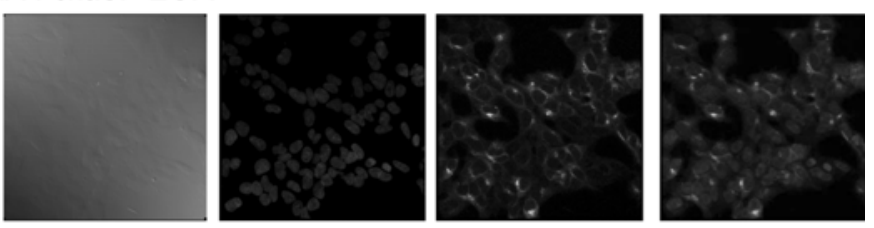

(C)

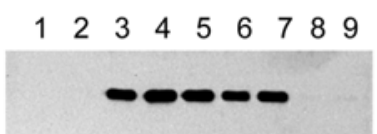

Fig. 5. Expression and secretion of an FcnaSP-EGFP fusion protein from HEK 293 cells. (A) Construction of FcnaSP-EGFP fusion protein expression vector. FcnaSP-EGFP gene fusion encoded a 17-aa FcnaSP signal sequence fused to full-length EGFP protein. (B) Expression of the FcnaSP-EGFP fusion protein. Expression of the FcnaSP-EGFP fusion protein was detected in the stable cell line by confocal microscopy. (C) Secretion of FcnaSP-EGFP fusion protein. Culture medium of the stable cell line transfected with FcnaSPEGFP fusion gene was loaded onto an Ni-NTA-agarose column. EGFP protein in eluted fractions was analyzed by Western blotting using antibody against Myc. 
secretion are targeted at translocation channels in the ER membrane, processed for vesicular transport in the Golgi apparatus, and then transported to the plasma membrane; signal sequences are then cleaved by signal peptidases (Gierash, 1989; Rothman and Wieland, 1996). Chimeric genes coding for signal peptides and useful proteins have been expressed in the mammalian and insect system to produce functional proteins. The main advantage of using a secreted protein system, in contrast with intracellular proteins, is that they produce post-translationally modified proteins.

Ficolin A is a plasma protein with lectin activity, and plays a role in host defense by binding carbohydrates, especially GlcNAc, on microorganisms. The ficolin A subunit consists of an N-terminal signal peptide, a collagen-like domain, and a C-terminal fibrinogen-like domain. A putative ficolin A signal peptide (MQWPTLWAFSGLLCLCPSQA) was predicted in a previous report (Fujimori et al., 1998). In the present study, we identified a functionally relevant signal peptide (MQWPTLWAFSGLLCLCP), and found in stable cell lines transfected with an expression vector encoding full length ficolin A that recombinant ficolin A is constitutively expressed and secreted into culture medium. The secreted protein was found to have an $\mathrm{N}$-terminal sequence, which suggested that a seventeen amino acid sequence (MQWPTLWAFSGLLCLCP) rather than a twenty amino acid sequence (MQWPTLWAFSGLLCLCPSQA) functions as a signal peptide. When mouse ficolin A signal peptide was fused with EGFP, EGFP was released into medium by HEK 293 cells. Therefore, we conclude that the new signal peptide sequence can drive protein secretion. Our results also suggest that the signal peptide of ficolin A has potential for producing useful secreted proteins.

Acknowledgments This work was supported by a Korean Research Foundation Grant funded by the Korean Government (MOEHRD, Basic Research Promotion Fund, KRF-2005070-C00091) and by the Korean Next Generation Growth Engine Program (\#F104AC010002-06A0301-00230). We thank Seung-Hae Kwon of the Korean Basic Science Institute Chuncheon Center for technical assistance with the confocal image analyses (LSM 510 META NLO).

\section{References}

Aderem, A. and Ulevitch, R. J. (2000) Toll-like receptors in the induction of the innate immune response. Nature 406, 782-787.

Dahl, M. R., Thiel, S., Matsushita, M., Fujita, T., Willis, A. C., Christensen, T., Vorup-Jensen, T. and Jensenius, J. C. (2001) MASP-3 and its association with distinct complexes of the mannan-binding lectin complement activation pathway. Immunity 15, 127-135.

Endo, Y., Nakazawa, N., Liu, Y., Iwaki, D., Takahashi, M., Fujita, T., Nakata, M. and Matsushita, M. (2005) Carbohydratebinding specificities of mouse ficolin A, a splicing variant of ficolin $\mathrm{A}$ and ficolin $\mathrm{B}$ and their complex formation with
MASP-2 and sMAP. Immunogenetics 57, 837-844.

Endo, Y., Sato, Y., Matsushita, M. and Fujita, T. (1996) Cloning and characterization of the human lectin P35 gene and its related gene. Genomics 36, 515-521.

Gierasch, L. M. (1989) Signal sequences. Biochemistry 28, 923930.

Fujimori Y., Harumiya, S., Fukumoto, Y., Miura, Y., Yagasaki, K., Tachikawa, H. and Fujimoto, D. (1998) Molecular cloning and characterization of mouse ficolin-A. Biochem. Biophys. Res. Commun. 244, 796-800.

Fujita, T. Matsushita, M. and Endo, Y. (2004) The lectincomplement pathway-its role in innate immunity and evolution. Immunol. Rev. 198, 185-202.

Holmskov, U., Thiel, S. and Jensenius, J. C. (2003) Collection and ficolins: humoral lectins of the innate immune defense. Аnпu. Rev. Immunol. 21, 547-578.

Ichijo, H., Hellman, U., Wernstedt, C., Gonez, L. J., ClaessonWelsh, L., Heldin, C. H. and Miyazono, K. (1993) Molecular cloning and characterization of ficolin, a multimeric protein with fibrinogen- and collagen-like domains. J. Biol. Chem. 268, 14505-14513.

Janeway, C. A. Jr. and Medzhitov, R. (1998) Introduction: the role of innate immunity in the adaptive immune response. Semin. Immunol. 10, 349-350.

Kwon, H. J., Lee, K. W., Yu, S. H., Han, J. H. and Kim, D. S.

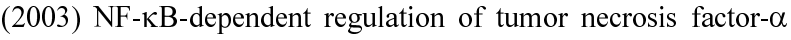
gene expression by CpG-oligodeoxynucleotides. Biochem. Biophys. Res. Commun. 311, 129-138.

Lee Y., Sohn, W. J., Kim, D. S. and Kwon, H. J. (2004) NF-кBand c-Jun-dependent regulation of human cytomegalovirus immediate-early gene enhuncer/promoter in response to lipopolysaccharie and bacterial CpG-oligodeoxynucleotides in macrophase cell in-RAW 264.7 Eur. J. Biochem. 271, 10941105.

Matsushita, M., Endo, Y., Taira, S., Sato, Y., Fujita, T., Ichikawa, N., Nakata, M. and Mizuochi, T. (1996) A novel human serum lectin with collagen- and fibrinogen-like domains that functions as an opsonin. J. Biol. Chem. 271, 2448-2454.

Matsushita, M. and Fujita, T. (1992) Activation of the classical complement pathway by mannose-binding protein in association with a novel C1s-like serine protease. J. Exp. Med. 176, 14971502.

Matsushita, M. and Fujita, T. (2001) Ficolins and the lectin complement pathway. Immunol. Rev. 180, 78-85.

Ohashi, T. and Erickson, H. P. (1998) Oligomeric structure and tissue distribution of ficolins from mouse, pig and human. Arch. Biochem. Biophys. 360, 223-232.

Rothman, J. E. and Wieland, F. T. (1996) Protein sorting by transport vesicles. Science 272, 227-234.

Roosi, V., Cseh, S., Bally, I., Thielens, N. M., Jensenius, J. C. and Arlaud, G. J. (2002) Substrate specificities of recombinant mannan-binding lectin-associated serine proteases-1 and -2. $J$. Biol. Chem. 276, 40880-40887.

Roos, A., Bouwman, L. H., Munoz, J., Zuiverloon, T., Faber-Krol, M. C., Fallaux-van den Houten, F. C., Klar-Mohamad, N., Hack, C. E., Tilanus, M. G. and Daha, M. R. (2003) Functional characterization of the lectin pathway of complement in human serum. Mol. Immunol. 39, 655-668.

Thiel, S., Vorup-Jensen, T., Stover, C. M., Schwaeble, W., Laursen, S. B., Poulsen, K., Willis, A. C., Eggleton, P., 
Hansen, S., Holmskov, U., Reid, K. B. and Jensenius, J. C. (1997) A second serine protease associated with mannanbinding lectin that activates complement. Nature 386, 506-510.

Vorup-Jensen, T., Petersen, S. V., Hansen, A. G., Poulsen, K., Schwaeble, W., Sim, R. B., Reid, K. B., Davis, S. J., Thiel, S. and Jensenius, J. C. (2000) Distinct pathways of mannanbinding lectin (MBL)- and C1-complex autoactivation revealed by reconstitution of MBL with recombinant MBL-associated serine protease-2. J. Immunol. 165, 2093-2100.

Schwaeble, W., Dahl, M. R., Thiel, S., Stover, C. M. and
Jensenius, J. C. (2002) The mannan-binding lectin-associated serine proteases (MASPs) and Map19: four components of the lectin pathway activation complex encoded by two genes. Immunobiology 205, 455-466.

Sugimoto, R., Yae, Y., Aikawa, M., Kitajima, S., Shibata, Y., Sato, H., Hirata, J., Okochi, K., Izuhara, K. and Hamasaki, N. (1998) Cloning and characterization of the Hakata antigen, a member of the ficolin/opsonin p35 lectin family. J. Biol. Chem. 273, 20721-20727. 